\title{
Composite fermions in Electroweak Symmetry Breaking
}

\author{
Riccardo Barbieri $^{a}$, Gino Isidori $^{a, b}$, Duccio Pappadopulo $^{a}$ \\ a Scuola Normale Superiore and INFN, Piazza dei Cavalieri 7, 56126 Pisa, Italy \\ ${ }^{b}$ INFN, Laboratori Nazionali di Frascati, Via E. Fermi 40 I-00044 Frascati, Italy
}

\begin{abstract}
If the electroweak symmetry is broken by some unspecified strong dynamics, composite fermions may exist with definite transformation properties under $S U(2)_{L} \times S U(2)_{R} / S U(2)_{L+R}$ and may play a role in giving masses by mixing to all the standard quarks and leptons. Assuming this to be the case, we analyze the role of Singlets, Doublets and Triplets in the ElectroWeak Precision Tests and in Flavour Physics. Doublets and Triplets are generically disfavoured. In the Singlet case, we specify the breaking patterns of the flavour group that allow to keep the CKM picture of flavour physics and we discuss the effects of the mixing between composite and elementary fermions. These mixings affect in particular the rather peculiar LHC phenomenology of the composite fermions.
\end{abstract}




\section{Introduction and statement of the framework}

That a perturbative Higgs boson exist, one or more and perhaps supersymmetric, is highly probable, with its elusiveness so far explained by the lack of direct experiments at the Fermi scale or above it. The difficulty of proposing explicit and sensible Higgsless models, despite some interesting recent attempts [1, 2, 3, is not the least reason behind this view. However, while waiting for the LHC to say the final word on this issue, we find useful to spend some time in exploring possible generic patterns of Higgsless descriptions of ElectroWeak Symmmetry Breaking (EWSB). In fact, it is again the very lack of direct experiments at the relevant energy scale that motivates us, since this lack may hide some clues needed to understand the physics of EWSB.

We base our considerations on the following generic picture. Some strong dynamics breaks a $S U(2)_{L} \times S U(2)_{R} \times U(1)_{X}$ symmetry, global in the limit of vanishing electroweak gauge couplings, down to $S U(2)_{L+R} \times U(1)_{X}$. This spontaneous symmetry breaking, characterised by the scale $v=\left(\sqrt{2} G_{F}\right)^{-1 / 2} \approx 246$, also leads to the breaking of the standard electroweak gauge symmetry, $S U(2)_{L} \times U(1)_{Y}, Y=T_{3 R}+X$, down to the electromagnetic $U(1)$. There exist three generations of fermions with the usual $S U(2)_{L} \times U(1)_{Y}$ quantum numbers, called elementary since they do not feel directly the strong dynamics. The strong dynamics produces composite vectors and composite fermions with definite transformation properties under $S U(2)_{L} \times S U(2)_{R} / S U(2)_{L+R}$. These nonlinear transformations involve as usual the Goldstone fields, $\hat{\pi}=\pi^{a} \sigma^{a} / 2$, themselves transforming under $S U(2)_{L} \times S U(2)_{R}$ as

$$
U \rightarrow g_{R} U g_{L}^{\dagger}, \quad U \equiv e^{i 2 \hat{\pi} / v} .
$$

The exchange of the composite vectors is supposed to keep unitary the scattering amplitudes of $W$ 's and $Z$ 's up to the scale $\Lambda \approx 4 \pi v \approx 3 \mathrm{TeV}$ and may give an important contribution to the ElectroWeak Precision Tests (EWPT), both at tree and loop level [4]. For the composite fermions, which are the focus of this work, we consider Singlets, Doublets or Triplets under $S U(2)_{L+R}$. They also contribute, at loop level, to the EWPT. Furthermore they carry a generation index $i=1,2,3$ as the standard elementary fermions and a $X$ quantum number that allows them to mix with the elementary fermions consistently with gauge invariance. We hope that the multiplicity of these states be somehow explained by the strong dynamics. A crucial assumption we make is that, in absence of this mixing, the elementary fermions are massless 1 and the strong dynamics does not distinguish the different generations. We shall show that a definite symmetry pattern for the mixing between the elementary and the composite fermions allows to keep, in the Singlet case, the CKM picture of flavour physics, although with some characteristic effects in flavour physics still emerging.

While not resting on any explicit model of EWSB, which may actually be difficult to exhibit at all with present knowledge, the interesting aspect of the picture outlined above is that some of its consequences can be analyzed on general grounds. Not surprisingly, this is based on the symmetries of the problem: on one side the chiral $S U(2)_{L} \times S U(2)_{R}$ spontaneously broken down to $S U(2)_{L+R}$ and, on the other side, the postulated flavour symmetry, $G_{f}$, of the strong dynamics and of the weak gauge interactions in absence of mixing between the composite and the elementary fermions.

\footnotetext{
${ }^{1}$ An early paper discussing the mass generation of the ordinary fermions by mixing with composite fermions is [5].
} 
The content of the paper is the following. In Section 2 we describe the effective chiral Lagrangians of the composite fermions up to one derivative terms, including the mass mixing with the elementary fermions. In Section 3 we discuss some of the effects of the composite Doublets or Triplets in the EWPT. We take these considerations as evidence for a generic difficulty of Doublets or Triplets in a truly strongly interacting theory of EWSB, unless some parameters are suitably adjusted. As shown in Section 4 , even considerations of flavour physics make Doublets and Triplets somewhat disfavoured. On the other hand, the effective Lagrangian for composite Singlets up to one derivative terms is identical to the one of elementary fermions with the same weak quantum numbers, thus screening at all their composite nature. They may play however an interesting role in Flavour Physics by mixing with the elementary fermions in a suitable way, as discussed in Section 4. We require that the flavour symmetry $G_{f}^{\mathcal{S}}$ be broken by mixing parameters which, treated as spurions, have definite transformation properties under $G_{f}^{\mathcal{S}}$. In the case of composite Singlets, this enables us to enforce a specific case of Minimal Flavour Violation, with the CKM matrix as the only control of flavour changing phenomena. Yet the mixing between the elementary and the composite singlets gives rise to significant residual effects both in the EWPT and in Flavour Physics, as discussed in Section 5. While all the discussion is concentrated on quarks, the picture is easily extended to leptons (See Section 6). The collider phenomenology is briefly described in Section 7 . The conclusions are summarized in Section 8.

\section{Effective Lagrangians}

We consider in turn the effective Lagrangians for Singlets, Doublets and Triplets under $S U(2)_{L+R}$ up to one derivative terms, including the general mass mixings with the elementary fermions. We concentrate on quarks, leaving the straightforward extension to leptons in Section 7 . As mentioned, the symmetry includes a $U(1)_{X}$ to allow the standard gauging of hypercharge.

Other than (1.1), the key ingredient to describe the transformation properties of the various fields under the full $S U(2)_{L} \times S U(2)_{R}$ is the little matrix $u$ [6] via

$$
U=u^{2} .
$$

This matrix parametrizes the $S U(2)_{L} \times S U(2)_{R} / S U(2)_{L+R}$ coset and transforms a: 2

$$
u \rightarrow g_{R} u h^{\dagger}=h u g_{L}^{\dagger},
$$

where $h=h\left(u, g_{L}, g_{R}\right)$ is uniquely determined by this equation. In turn, important functions of $u$ are

$$
\Gamma_{\mu}=\frac{1}{2}\left[u^{\dagger}\left(\partial_{\mu}-i \hat{B}_{\mu}\right) u+u\left(\partial_{\mu}-i \hat{W}_{\mu}\right) u^{\dagger}\right], \quad \Gamma_{\mu}^{\dagger}=-\Gamma_{\mu}, \quad \Gamma_{\mu} \rightarrow h \Gamma_{\mu} h^{\dagger}+h \partial_{\mu} h^{\dagger},
$$

and

where

$$
u_{\mu}=i u^{\dagger} D_{\mu} U u^{\dagger}=u_{\mu}^{\dagger}, \quad u_{\mu} \rightarrow h u_{\mu} h^{\dagger}
$$

$$
D_{\mu} U=\partial_{\mu} U-i \hat{B}_{\mu} U+i U \hat{W}_{\mu}, \quad \hat{W}_{\mu}=g T^{a} W_{\mu}^{a}, \quad \hat{B}_{\mu}=g^{\prime} T^{3} B_{\mu} .
$$

\footnotetext{
${ }^{2}$ Note that here as in [4] we follow the convention, usually adopted in QCD, where the $L$-transformations act on the right and viceversa. This is unlike what is normally done in discussing strong EWSB.
} 


\section{$2.1 \quad$ Singlets}

Singlets under $S U(2)_{L+R}$ that can mix with the standard quarks are $U$ and $D$, carrying colour and $X$-numbers $2 / 3$ and $-1 / 3$ respectively. Their covariant kinetic term is the trivial one and no other one-derivative term appears $\left(\operatorname{Tr}\left(u_{\mu}\right)=0\right)$. In absence of mixing with the elementary fermions, the interactions of the singlets $\mathcal{S}$ with the Goldstone fields appear only at two-derivative level and are suppressed by inverse powers of the cutoff. Their composite nature may be difficult to see directly. They may however play an interesting role in giving masses by mixing to the standard fermions. Introducing the fictitious doublets, one per generation,

$$
Q \equiv\left(\begin{array}{c}
U \\
D
\end{array}\right)
$$

the most general mixing mass term, including the necessary $S U(2)_{R}$ breaking, i. 3

$$
L_{m i x}^{\mathcal{S}}=m_{L}^{u} \bar{Q}_{R} \hat{P}_{u} U q_{L}+m_{R}^{u} \bar{Q}_{L} \hat{P}_{u} q_{R}+m_{L}^{d} \bar{Q}_{R} \hat{P}_{d} U q_{L}+m_{R}^{d} \bar{Q}_{L} \hat{P}_{d} q_{R}+\text { h.c. }
$$

where $\hat{P}_{u(d)}=\left(1 \pm \sigma_{3}\right) / 2$. All these $m$ 's are matrices in flavour space. In absence of mixing with the elementary fermions, the composite singlets have flavour independent masses:

$$
L_{\mathcal{S}}=i \bar{Q} \gamma^{\mu}\left(\partial_{\mu}-i g^{\prime} X B_{\mu}\right) Q+M_{U} \bar{U} U+M_{D} \bar{D} D
$$

A discussion about the flavour structure of the model is postponed to Section 4 and 5. Here we limit to note that in the mass-eigenstate basis the relation between the mass parameters in (2.5) $-(2.6)$ and the physical masses of light and heavy states are given by Eqs. (5.2)-(5.4).

\subsection{Doublets}

The Doublets

$$
\mathcal{D} \equiv\left(\begin{array}{c}
T \\
B
\end{array}\right)
$$

of $X$-number $1 / 6$, transform under $S U(2)_{L+R}$ as $\mathcal{D} \rightarrow h \mathcal{D}$. We call the up and down components of $\mathcal{D}$ respectively $T$ and $B$, not to confuse them with the $S U(2)_{L+R}$ singlets with the same charge, $U$ and $D$. The most general invariant Lagrangian up to one-derivative terms is

$$
L_{\mathcal{D}}=i \overline{\mathcal{D}} \gamma^{\mu}\left(\partial_{\mu}+\Gamma_{\mu}-i g^{\prime} X B_{\mu}\right) \mathcal{D}+\frac{\alpha}{2} \overline{\mathcal{D}} \gamma_{\mu} \gamma_{5} u_{\mu} \mathcal{D}+M_{\mathcal{D}} \overline{\mathcal{D}} \mathcal{D}
$$

The strong dynamics is assumed to conserve parity.

The mixing Lagrangian is

$$
L_{\text {mix }}^{\mathcal{D}}=m_{L}^{u} \overline{\mathcal{D}}_{R} u^{\dagger} \hat{P}_{u} U q_{L}+m_{R}^{u} \overline{\mathcal{D}}_{L} u^{\dagger} \hat{P}_{u} q_{R}+m_{L}^{d} \overline{\mathcal{D}}_{R} u^{\dagger} \hat{P}_{d} U q_{L}+m_{R}^{d} \overline{\mathcal{D}}_{L} u^{\dagger} \hat{P}_{d} q_{R}+\text { h.c. }
$$

whose invariance follows from $u^{\dagger} \mathcal{D} \rightarrow g_{L} u^{\dagger} \mathcal{D}$ and $u \mathcal{D} \rightarrow g_{R} u \mathcal{D}$. Neglecting terms containing pions,

$$
i \Gamma_{\mu} \rightarrow \frac{1}{2}\left(\hat{W}_{\mu}+\hat{B}_{\mu}\right), \quad u_{\mu} \rightarrow \hat{B}_{\mu}-\hat{W}_{\mu} .
$$

\footnotetext{
${ }^{3}$ The $U$ appearing in all the $L_{m i x}$ of this Section is the matrix in (1.1) and should not be confused with the heavy fermion $U$.
} 
Therefore the electroweak interactions of the Doublets are

$$
L_{\mathcal{D}}^{i n t}=\overline{\mathcal{D}} \gamma^{\mu}\left[\hat{W}_{\mu} \frac{1}{2}\left(1-\alpha \gamma_{5}\right)+\hat{B}_{\mu} \frac{1}{2}\left(1+\alpha \gamma_{5}\right)+g^{\prime} X B_{\mu}\right] \mathcal{D}
$$

(which reduce to the ones of an elementary quark family for $\alpha=1$ ).

\subsection{Triplets}

The Triplets $\mathcal{T}$ that contain bot an up and a down-type quark have $X=2 / 3$ or $X=-1 / 3$ and transform under $S U(2)_{L+R}$ as $\mathcal{T} \rightarrow h \mathcal{T} h^{+}$. In $2 \times 2$ matrix notation, e.g. for $X=2 / 3$, it is

$$
\mathcal{T}=\left(\begin{array}{cc}
T / \sqrt{2} & X^{5 / 3} \\
B & -T / \sqrt{2}
\end{array}\right)
$$

where $X^{5 / 3}$ is an exotic quark of charge $2 / 3$.

The most general invariant Lagrangian is

$$
L_{\mathcal{T}}=i \operatorname{Tr}\left[\overline{\mathcal{T}} \gamma^{\mu}\left(\partial_{\mu} \mathcal{T}+\left[\Gamma_{\mu}, \mathcal{T}\right]-i g^{\prime} X B_{\mu} \mathcal{T}\right)\right]+\alpha \operatorname{Tr}\left[\overline{\mathcal{T}} \gamma_{\mu} \gamma_{5} u_{\mu} \mathcal{T}\right]+M_{\mathcal{T}} \overline{\mathcal{T}} \mathcal{T}
$$

with the electroweak gauge interactions that reduce to

$$
L_{\mathcal{T}}^{i n t}=\operatorname{Tr}\left[\overline{\mathcal{T}} \gamma^{\mu}\left(\hat{W}_{\mu}\left(1-\alpha \gamma_{5}\right)+\hat{B}_{\mu}\left(1+\alpha \gamma_{5}\right)+g^{\prime} X B_{\mu}\right) \mathcal{T}\right]
$$

To construct the mixing Lagrangian, note that, under $S U(2)_{L} \times S U(2)_{R}$

$$
\begin{aligned}
u^{\dagger} \mathcal{T} u^{\dagger} & \rightarrow g_{L}\left(u^{\dagger} \mathcal{T} u^{\dagger}\right) g_{R}^{\dagger} \\
u \mathcal{T} u^{\dagger} & \rightarrow g_{R} R\left(u \mathcal{T} u^{\dagger}\right) g_{R}^{\dagger} .
\end{aligned}
$$

Defining the vectors

$$
\left(u \overline{\mathcal{T}} u^{\dagger}\right)_{1 i}=\bar{v}_{i}, \quad(u \overline{\mathcal{T}} u)_{1 i}=\bar{w}_{i}
$$

the mixing Lagrangian is

$$
L_{\text {mix }}^{\mathcal{T}}=m_{L}^{u} \bar{w}_{R} U^{\dagger} \hat{P}_{u} U q_{L}+m_{R}^{u} \bar{v}_{L} \hat{P}_{u} q_{R}+m_{L}^{d} \bar{w}_{R} U^{\dagger} \hat{P}_{d} U q_{L}+m_{R}^{d} \bar{v}_{L} \hat{P}_{d} q_{R}+\text { h.c. }
$$

\section{$3 \quad$ EWPT for Doublets and Triplets}

\section{$3.1 \Delta S$}

Even in absence of any mixing, the Doublets and the Triplets contribute to the the EWPT through the $S$-parameter. Each doublet and triplet contribute respectively to the $S$-parameter as

$$
\begin{aligned}
\Delta S^{(\mathcal{D})} & =\frac{1}{2 \pi}\left[1-\left(\alpha^{2}-1\right)\left(\log \Lambda^{2} / M_{\mathcal{D}}^{2}\right)\right] \\
\Delta S^{(\mathcal{T})} & =\frac{2}{\pi}\left[1-\left(\alpha^{2}-1\right)\left(\log \Lambda^{2} / M_{\mathcal{T}}^{2}\right)\right]
\end{aligned}
$$


where $\Lambda$ is a suitable UV cutoff. Especially if there is one such contribution per generation, this is a pretty large effect (which might be negative if $\alpha>1$ ). This $\Delta S$ is reminiscent of the well known contribution from technifermions in TechniColour. In our "effective" view, there is one contribution as in (3.1) or (3.2) per generation and we would have to add it to the contribution from heavy vectors, which occurs at tree level.

Eq.s (3.1) or (3.2) appear to us as a generic difficulty for a truly strongly interacting theory of EWSB. It is of some interest, however, to make contact with models in the literature that have a moderate or even vanishing $\Delta S$ from fermion loops. One case is if the composites, either doublets or triplets, occur in full representations of $S U(2)_{L} \times S U(2)_{R}$ before symmetry breaking. This is meaningful only if the compositeness scale is higher than the scale of electroweak symmetry breaking, like in models where there is a Higgs doublet, either elementary or composite. Examples are vector-like representations $(2,1) \oplus(1,2)$ or $(2,2)$ of $S U(2)_{L} \times S U(2)_{R}$ with suitable $X$-numbers, giving respectively 2 doublets $\left(\mathcal{D}_{1}, \mathcal{D}_{2}\right)$ or one triplet and one singlet $(\mathcal{T} \oplus S)$ under $S U(2)_{L+R}$. In this case, $\Delta S$ may vanish. As easily seen by explicit calculations, this requires degeneracy of the full representation after $S U(2)_{L} \times S U(2)_{R} \rightarrow S U(2)_{L+R}$ breaking and perturbative uncorrected gauge couplings, which speaks against a strongly interacting composite Higgs boson.

Another example of formally vanishing $\Delta S$ from fermion loops comes from a Dirac fermion transforming as $(1,2,1)$ under $S U(2)_{L} \times S U(2)_{C} \times S U(2)_{R}$, broken down to the diagonal subgroup, $S U(2)_{L+C+R}$, like in the so called Three-site Model [7]. This requires that the mass of the doublet be much larger than the masses of the vector bosons and that perturbation theory makes sense in all the couplings, including the coupling $g_{C}$ of $S U(2)_{C}$. In this case, (3.1) remains formally correct, with $\alpha=0$, but the full $\Delta S$ from the fermionic loop is reabsorbed, after renormalization of $g_{C}$, in the tree level effect to $S$ due to the kinetic mixing of the vector bosons.

\section{$3.2 \Delta T$ and $Z \rightarrow \bar{b} b$}

Unlike $\Delta S$, a contribution to the $T$ parameter arises only after breaking of the custodial symmetry from the composite/elementary mixing, which is important from the third family only. In fact, in presence of a strong breaking of this symmetry in the left-handed sector, i.e. $m_{L}^{d}<<m_{L}^{u}$, explicit calculations show that $\Delta T$ is always unacceptably large, whereas it is moderate and generally positive, if $m_{L}^{u} \approx m_{L}^{d}$, so as to minimize custodial breaking. In this case, however, it is crucial to watch the $Z \bar{b}_{L} b_{L}$ coupling, because of the $b_{L} / B_{L}$-mixing that occurs at tree level.

Let us consider the deviations from the SM of the $Z \bar{b} b$ couplings, $\delta g_{L}, \delta g_{R}$. It is in general (an overall factor $g / c_{W}$ taken away)

$$
\delta g_{L, R}=\left(s_{L, R}^{b}\right)^{2}\left[g_{L, R}(B)-g_{L, R}(b)\right]
$$

where $s_{L, R}^{b}$ are the sines of the mixing angles in the down sector for the third generation. From the Lagrangians of Eq.s (2.11) and (2.13) one gets respectively:

- Doublet case

$$
\delta g_{L}=\frac{\left(s_{L}^{b}\right)^{2}}{4}(1-\alpha), \quad \delta g_{R}=-\frac{\left(s_{R}^{b}\right)^{2}}{4}(1-\alpha)
$$


- Triplet case

$$
\delta g_{L}=-\frac{\left(s_{L}^{b}\right)^{2}}{2} \alpha, \quad \delta g_{R}=-\frac{\left(s_{R}^{b}\right)^{2}}{2}(1-\alpha) .
$$

Now for generic $\alpha=O(1)$ and $m_{L}^{d} \approx m_{L}^{u}$ for the third generation, this is a strong constraint. In fact, from the diagonalization of the mass matrix of the third generation (see Section 5) one finds

$$
s_{L}^{b} \approx \frac{m_{t}}{M_{T}} \frac{1}{s_{R}^{t} c_{R}^{t}}, \quad s_{R}^{b} \approx \frac{m_{b}}{m_{t}} s_{R}^{t}
$$

where $s_{R}^{t}$ and $c_{R}^{t}$ denote sine and cosine of the mixing angle of the right-handed top with its composite partner of mass $M_{T}$. Whatever the value of $s_{R}^{t}$ is, these equations make $\delta g_{R}$ irrelevant (i.e. no explanation offered for the notorious problem of the $b$ forward-backward asymmetry) whereas, depending on the value of $\alpha$, a strong bound on $M_{T}$ generally arises from the contribution of $\delta g_{L}$ to $\Gamma(Z \rightarrow \bar{b} b)$.

From the composite/elementary mixing also the Singlets contribute to $\Delta T$ and to $Z \rightarrow \bar{b} b$. We shall come back to this in Section 5 .

\section{Flavour symmetries}

As mentioned in the Introduction we assume that, in absence of composite/elementary mixing, the system possesses a large flavour symmetry which extends the one of the SM for vanishing Yukawa couplings,

$$
G_{f}^{S M}=S U(3)_{q} \times S U(3)_{u R} \times S U(3)_{d R}
$$

to include also the flavour symmetry of the composite sector, i.e.

$$
G_{f}^{\mathcal{S}}=S U(3)_{U} \times S U(3)_{D} \times S U(3)_{q} \times S U(3)_{u R} \times S U(3)_{d R}
$$

or

$$
G_{f}^{\mathcal{D}, \mathcal{T}}=S U(3)_{\mathcal{D}, \mathcal{T}} \times S U(3)_{q} \times S U(3)_{u R} \times S U(3)_{d R} .
$$

Both in the SM viewed as an effective theory or here, these flavour symmetries have to be broken appropriately to keep consistency with experiments. An additional problem of the flavour-breaking mixing terms in the effective Lagrangians in Section 2, if treated generically, is the large number of physical parameters, even increased relative to the SM.

\subsection{Minimal Flavour Violation}

As well known, the SM model viewed as an effective theory gives a consistent description of flavour physics, provided the flavour group in the quark sector, $G_{f}^{S M}$, is only broken by two dimensionless parameters, $Y^{u}$ and $Y^{d}$, which, treated as spurions, transform as

$$
Y^{u}=(3, \overline{3}) \quad \text { under } \quad S U(3)_{u R} \times S U(3)_{q}
$$

\footnotetext{
${ }^{4} \delta g_{L}=0$ or $\alpha=0$ in the Triplet case results if the Triplet comes from a $(2,2)$ of $S U(2)_{L} \times S U(2)_{R}$ and the perturbative gauge couplings are kept uncorrected [8].
} 


$$
Y^{d}=(3, \overline{3}) \quad \text { under } \quad S U(3)_{d R} \times S U(3)_{q}
$$

Such an hypothesis enforces in particular the successful CKM picture, since, without loss of generality, $Y^{d}$ can be reduced to diagonal form, $Y^{d}=\lambda^{d}$, and similarly $Y^{u}$ can be diagonalized up to a single unitary matrix, $Y^{u}=\lambda^{u} V$, where $V$ is the CKM matrix. As long as this symmetry and this symmetry breaking pattern is respected, even the inclusion of higher dimensional operators, suppressed by a scale of $3 \div 5 \mathrm{TeV}$, is harmless [9].

\subsection{Singlets}

It would be nice if the above picture of flavour physics could be extended to the situation we are considering here, keeping in particular under control the number of new parameters in the flavour sector. This is in fact neatly the case under one of the following circumstances for the flavour group $G_{f}^{\mathcal{S}}$, that we name Parity Conserving $(P C)$ and Parity Breaking (PB):

- 1. Parity Conserving

$G_{f}^{\mathcal{S}}$ is only broken by

$$
\begin{array}{lll}
Y_{1}^{u}=(3, \overline{3}) & \text { under } & S U(3)_{U} \times S U(3)_{q+u R} \\
Y_{1}^{d}=(3, \overline{3}) & \text { under } & S U(3)_{D} \times S U(3)_{q+d R}
\end{array}
$$

where $S U(3)_{q+u R}$ or $S U(3)_{q+d R}$ denote the corresponding diagonal groups. For the mass matrices in Eq. (2.5) this implies:

$$
m_{L}^{u}=v Y_{1}^{u}, \quad m_{R}^{u}=f^{u} Y_{1}^{u}, \quad m_{L}^{d}=v Y_{1}^{d}, \quad m_{R}^{d}=f^{d} Y_{1}^{d},
$$

where $f^{u}, f^{d}$ are two mass scales, likely of the same order as the composite quark masses, $M_{U}, M_{D}$ in (2.6), and $V$ is again the CKM matrix. After diagonalization of $Y_{1}^{u}$ and $Y_{1}^{d}$ and suitable redefinitions of the various fields in generation space, the overall mass Lagrangian can be written as

$$
L_{m i x}^{\mathcal{S}}(P C)=v \bar{U}_{R} \lambda^{u} V u_{L}+f^{u} \bar{U}_{L} \lambda^{u} u_{R}+v \bar{D}_{R} \lambda^{d} d_{L}+f^{d} \bar{D}_{L} \lambda^{d} d_{R}+\text { h.c. }
$$

with diagonal $\lambda^{u}$ and $\lambda^{d}$.

- 2. Parity Breaking

$G_{f}^{\mathcal{S}}$ is broken down to $S U(3)_{U+u R} \times S U(3)_{D+d R} \times S U(3)_{q}$, which is in turn only broken by

$$
\begin{array}{lll}
Y_{2}^{u}=(3, \overline{3}) & \text { under } & S U(3)_{U+u R} \times S U(3)_{q} \\
Y_{2}^{d}=(3, \overline{3}) & \text { under } & S U(3)_{D+d R} \times S U(3)_{q},
\end{array}
$$

so that in this case

$$
m_{L}^{u}=v Y_{2}^{u}, \quad m_{R}^{u}=f^{u} \mathbf{1}, \quad m_{L}^{d}=v Y_{2}^{d}, \quad m_{R}^{d}=f^{d} \mathbf{1} .
$$

The mass Lagrangian can be written as

$$
L_{m i x}^{\mathcal{S}}(P B)=v \bar{U}_{R} \lambda^{u} V u_{L}+f^{u} \bar{U}_{L} u_{R}+v \bar{D}_{R} \lambda^{d} d_{L}+f^{d} \bar{D}_{L} d_{R}+\text { h.c. }
$$


Note that in both cases the direct mass terms between two elementary fermions is not compatible with the required symmetry and symmetry breaking.

A third case analogous to 2 above with $G_{f}^{\mathcal{S}}$ broken down to $S U(3)_{U+q} \times S U(3)_{D+q} \times S U(3)_{u R} \times$ $S U(3)_{d R}$ would imply

$$
m_{L}^{u} \propto 1, \quad m_{R}^{u}=f^{u} Y^{u}, \quad m_{L}^{d} \propto 1, \quad m_{R}^{d}=f^{d} Y^{d} .
$$

Although also leading to a case where flavour mixing is controlled only by the CKM matrix, this case is not compatible with observations unless the mass $M_{U}$ of the composite $U$-quarks is taken well above $5 \mathrm{TeV}$. This is due to the intra-generation mixing of the left-handed light quarks, which spoils the precise tests of CKM unitarity (see e.g. Ref. [10]).

\subsection{Doublets and Triplets}

It is of interest to ask if suitable symmetry conditions, analogous to the previous ones, can force the CKM picture of flavour physics also in the case of composite doublets or triplets. The answer is nd5. The overall flavour symmetry now is $G_{f}^{\mathcal{D}, \mathcal{T}}$ in (4.3). Therefore, with reference, e.g., to the $\mathrm{PC}$ case above, the analogous condition is that $G_{f}^{\mathcal{D}}, \mathcal{T}$ be only broken by

$$
\begin{array}{rll}
Y_{3}^{u} & =(3, \overline{3}) \quad \text { under } & S U(3)_{\mathcal{D}, \mathcal{T}} \times S U(3)_{q+u R} \\
Y_{3}^{d}=(3, \overline{3}) & \text { under } & S U(3)_{\mathcal{D}, \mathcal{T}} \times S U(3)_{q+d R}
\end{array}
$$

In turn, by suitable redefinitions of the various fields, the mixing Lagrangian can be written as

$$
L_{\text {mix }}^{\mathcal{D}, \mathcal{T}}(P C)=v \bar{U}_{R} \mathcal{V} \lambda^{u} V u_{L}+f^{u} \bar{U}_{L} \lambda^{u} u_{R}+v \bar{D}_{R} \lambda^{d} d_{L}+f^{d} \bar{D}_{L} \lambda^{d} d_{R}+\text { h.c. }
$$

where $\mathcal{V}$ is a further unitary matrix that cannot be rotated away from the overall Lagrangian.

\subsection{Flavour breaking by higher dimensional operators}

As in the case without composite fermions, we have to ask if higher dimensional operators consistent with the symmetries and the symmetry breaking described above are compatible with observations. There are two sets of flavour-changing neutral-current (FCNC) dimension-six operators, weighted by the inverse square of the cutoff and by suitable dimensionless coefficients, $c_{i j}$, $i, j=1,2,3$ :

$$
O_{L L}^{i j}=\left(\bar{q}_{L}^{i} \gamma_{\mu} q_{L}^{j}\right)^{2}, \quad O_{L R}^{i j}=\left(\bar{q}_{R}^{i} \hat{P}_{d} U\left[\gamma_{\mu}, \gamma_{\nu}\right] q_{L}^{j}\right) B_{\mu \nu}
$$

which are most significant. In Table 1 we give the expected expressions for the $c_{i j}$ in the Singlet case according to the symmetry breaking conditions formulated in Section 4.2 and we compare them with the analogous expressions obtained in MFV [9]. They are numerically equivalent if $s_{R}^{t}$ is of order unity, showing that a cutoff scale of about $3 \div 5 \mathrm{TeV}$ is also in this case compatible with current data.

\footnotetext{
${ }^{5}$ As in the singlet case we discard here as well, and for the same reason, the symmetry $S U(3)_{\mathcal{D}+q} \times S U(3)_{u R} \times$ $S U(3)_{d R}$.
} 


\begin{tabular}{|c||c|c|c|}
\hline Operators & MFV & Singlets PC & Singlets PB \\
\hline \hline$\frac{c_{i j}}{\Lambda^{2}} O_{L L}^{i j}$ & $c_{i j}=\left(Y^{u \dagger} Y^{u}\right)_{i j}^{2}$ & $c_{i j}=\left(Y_{1}^{u \dagger} Y_{1}^{u}\right)_{i j}^{2}$ & $c_{i j}=\left(Y_{2}^{u \dagger} Y_{2}^{u}\right)_{i j}^{2}$ \\
$(\Delta F=2)$ & $\approx \frac{m_{t}^{4}}{v^{4}}\left(V_{3 i}^{*} V_{3 j}\right)^{2}$ & $\approx \frac{m_{t}^{2}}{v^{2}} \frac{M_{T}^{2}}{\left(f^{u}\right)^{2}}\left(V_{3 i}^{*} V_{3 j}\right)^{2}$ & $\approx \frac{m_{t}^{4}}{v^{4}} \frac{M_{T}^{4}}{\left(f^{u}\right)^{4}}\left(V_{3 i}^{*} V_{3 j}\right)^{2}$ \\
\hline$\frac{c_{i j}}{\Lambda^{2}} O_{L R}^{i j}$ & $c_{i j}=\left(Y^{d} Y^{u \dagger} Y^{u}\right)_{i j}$ & $c_{i j}=\frac{f^{d}}{\Lambda}\left(Y_{1}^{d \dagger} Y_{1}^{d} Y_{1}^{u \dagger} Y_{1}^{u}\right)_{i j}$ & $c_{i j}=\frac{f^{d}}{\Lambda}\left(Y_{2}^{d} Y_{2}^{u \dagger} Y_{2}^{u}\right)_{i j}$ \\
$(\Delta F=1)$ & $\approx \frac{m_{d_{i}} m_{t}^{2}}{v^{3}} V_{3 i}^{*} V_{3 j}$ & $\approx \frac{m_{d_{i}} m_{t}}{v^{2}} \frac{M_{T} M_{D}}{f^{u} \Lambda} V_{3 i}^{*} V_{3 j}$ & $\approx \frac{m_{d_{i}} m_{t}^{2}}{v^{3}} \frac{M_{T}^{2} M_{D}}{\left(f^{u}\right)^{2} \Lambda} V_{3 i}^{*} V_{3 j}$ \\
\hline
\end{tabular}

Table 1: Comparison of the coefficients of the leading dimension-six operators relevant to $\Delta F=2$ and $\Delta F=1 \mathrm{FCNC}$ transitions of down-type quarks.

The situation is different in the Doublet or Triplet cases. Following the discussion in Section 4.3, a most dangerous effective Lagrangian is

$$
\Delta L_{\mathcal{D}, \mathcal{T}}^{\Delta F=2}=\frac{f_{d}}{\Lambda^{3}}\left(\bar{q}_{R} \hat{P}_{d} Y_{3}^{d \dagger} Y_{3}^{u} U q_{L}\right)^{2} \rightarrow \frac{m_{d} m_{c}\left(\mathcal{V}_{12}\right)^{2}}{v^{2}} \frac{M_{D} M_{U}}{f^{u} \Lambda^{3}}\left(\bar{d}_{R} s_{L}\right)^{2}
$$

where in the last step we have selected the $\Delta S=2$ contribution and $m_{d}, m_{c}$ are the masses of the down and charmed quarks. The matrix element of this effective Lagrangian between neutral kaons, for $\left|\mathcal{V}_{12}^{2}\right| \approx 1$, is about 100 times bigger than the matrix element of the leading dimensionsix $\Delta S=2$ operator in MFV. This issue (and a similar difficulty in $\Delta S=1$ left-right operators) is a manifestation of a general problem of composite models [11]: it appears in all cases where the suppression of FCNCs is attributed only to the small mixing of SM fermions and heavy states, but there is no flavour alignment between light and heavy states (see e.g. [12]).

\section{Composite/elementary mixing effects for Singlets}

\subsection{Tree level}

After sending $u_{L} \rightarrow V^{\dagger} u_{L}$, both the up and down mass matrices reduce to three $2 \times 2$ blocks, each labelled by a generation index $i$ :

$$
M_{i}^{(u)}=\left(\begin{array}{cc}
0 & f^{u} \lambda_{i}^{u}\left[f^{u}\right] \\
v \lambda_{i}^{u} & m_{U}
\end{array}\right), \quad M_{i}^{(d)}=\left(\begin{array}{cc}
0 & f^{d} \lambda_{i}^{d}\left[f^{d}\right] \\
v \lambda_{i}^{d} & m_{D}
\end{array}\right),
$$

where the values outside/within square brackets correspond to the PC/PB cases of Section 4.2. Relative to the SM, this introduces two extra parameters for each quark, which can be chosen as the mass of the heavy partner and the mixing angle in the left-handed or in the right-handed sector. In the limit where we neglect light quark masses, the corresponding left-handed mixing angles can be set to zero and the heavy states decouples in low-energy observables. 
Considering a generic $2 \times 2$ block, and defining left and right mixing angles as follows,

$$
\left(\begin{array}{cc}
-c_{R}^{q} & s_{R}^{q} \\
s_{R}^{q} & c_{R}^{q}
\end{array}\right)\left(\begin{array}{cc}
0 & m_{R}^{q} \\
m_{L}^{q} & M_{Q}
\end{array}\right)\left(\begin{array}{cc}
c_{L}^{q} & s_{L}^{q} \\
-s_{L}^{q} & c_{L}^{q}
\end{array}\right)=\operatorname{diag}\left(m_{q}, M_{Q_{q}}\right)
$$

we have ( $q$ stands for $u$ and $d$ and we omit for simplicity the index $q$ on the r.h.s.):

$$
\begin{aligned}
t_{R(L)}^{q} & =\frac{s_{R(L)}^{q}}{c_{R(L)}^{q}}=\frac{m_{R(L)}^{2}-m_{L(R)}^{2}-M_{Q}^{2}+\sqrt{M_{Q}^{4}+2 M_{Q}^{2}\left(m_{L}^{2}+m_{R}^{2}\right)+\left(m_{R}^{2}-m_{L}^{2}\right)^{2}}}{2 m_{R(L)} M_{Q}} \\
M_{Q_{q}}^{2}\left(m_{q}^{2}\right) & =\frac{M_{Q}^{2}+m_{R}^{2}+m_{L}^{2} \pm \sqrt{M_{Q}^{4}+2 M_{Q}^{2}\left(m_{L}^{2}+m_{R}^{2}\right)+\left(m_{R}^{2}-m_{L}^{2}\right)^{2}}}{2}
\end{aligned}
$$

which in the limit $m_{L}^{q} \ll M_{Q}$ reduce to

$$
M_{Q_{q}} \approx \sqrt{M_{Q}^{2}+\left(m_{R}^{q}\right)^{2}}, \quad m_{q} \approx \frac{m_{L}^{q} m_{R}^{q}}{M_{Q_{q}}}, \quad s_{R}^{q} \approx \frac{m_{R}^{q}}{M_{Q_{q}}}, \quad s_{L}^{q} \approx \frac{m_{L}^{q}}{M_{Q_{q}}} c_{R}^{q} .
$$

The results in Eq. (5.2)-(5.4) are completely general (they holds also for Doublets and Triplets in the appropriate mass-eigenstate basis). In the two specific cases discussed in Section 4.2 they imply

$$
\left.\left(s_{L}^{q}\right)^{2}\right|_{\mathrm{PC}} \approx \frac{v m_{q} M_{Q}^{2}}{f^{q} M_{Q_{q}}^{3}} \quad \text { or }\left.\quad\left(s_{L}^{q}\right)^{2}\right|_{\mathrm{PB}} \approx \frac{m_{q}^{2} M_{Q}^{2}}{\left(f^{q}\right)^{2} M_{Q_{q}}^{2}} .
$$

Since the two right-handed fields have the same quantum numbers, the rotation in the righthanded sector does not lead to observable effects and we can eliminate it by means of the exact relation

$$
t_{R}^{q} t_{L}^{q}=\frac{m_{q}}{M_{Q_{q}}} .
$$

Both in the PC and in the PB case the right-handed mixing in the top sector can be large if $f^{u} \sim M_{U}$. As we have seen in Section 4.4 this configuration is required for a natural suppression of the dimensions-six FCNC effective operators. We shall similarly assume $f^{d} \sim M_{D}$.

The rotation to the mass eigenstates leads to modifications in the interaction part of the SM Lagrangian. The couplings of light and heavy fermions to Goldstone bosons, $W$ and $Z$ fields (left-handed component) can be obtained from the SM Lagrangian with the replacements

$$
q_{L}^{i} \rightarrow c_{L}^{i} q_{L}^{i}+s_{L}^{i} Q_{L}^{i}, \quad m_{i} q_{R}^{i} \rightarrow m_{i} c_{L}^{i} q_{L}^{i}+M_{Q_{i}} s_{L}^{i} Q_{L}^{i}
$$

For instance, the currents coupled to $W$ and $Z$ fields, written in terms of the mass eigenstates, are

$$
\begin{aligned}
J_{W}^{\mu}= & \frac{g}{\sqrt{2}} \sum_{i j=1,3} V_{i j}\left[c_{L}^{u_{i}} c_{L}^{d_{j}} \bar{u}_{L}^{i} \gamma^{\mu} d_{L}^{j}+s_{L}^{u_{i}} s_{L}^{d_{j}} \bar{U}_{L}^{i} \gamma^{\mu} D_{L}^{j}+c_{L}^{u_{i}} s_{L}^{d_{j}} \bar{u}_{L}^{i} \gamma^{\mu} D_{L}^{j}+s_{L}^{u_{i}} c_{L}^{d_{j}} \bar{U}_{L}^{i} \gamma^{\mu} d_{L}^{j}\right] \\
J_{Z}^{\mu}= & \frac{g}{c_{W}} \sum_{i=1,6}\left\{\left(T_{3}\right)_{i}\left[\left(c_{L}^{i}\right)^{2} \bar{q}_{L}^{i} \gamma^{\mu} q_{L}^{i}+\left(s_{L}^{i}\right)^{2} \bar{Q}_{L}^{i} \gamma^{\mu} Q_{L}^{i}+s_{L}^{i} c_{L}^{i} \bar{q}_{L}^{i} \gamma^{\mu} Q_{L}^{i}+s_{L}^{i} c_{L}^{i} \bar{Q}_{L}^{i} \gamma^{\mu} q_{L}^{i}\right]\right. \\
& \left.-Q_{i} s_{W}^{2}\left[\bar{q}^{i} \gamma^{\mu} q^{i}+\bar{Q}^{i} \gamma^{\mu} Q^{i}\right]\right\},
\end{aligned}
$$


while the fermion couplings to a single Goldstone boson field are

$$
\begin{aligned}
\delta L_{\pi} & =\frac{\sqrt{2} i \pi^{+}}{v} \sum_{i j=1,3} V_{i j}\left[c_{L}^{u_{i}} m_{u_{i}} \bar{u}_{R}^{i}+s_{L}^{u_{i}} M_{U_{i}} \bar{U}_{R}^{i}\right]\left[c_{L}^{d_{j}} d_{L}^{j}+s_{L}^{d_{j}} D_{L}^{j}\right] \\
& +\frac{\sqrt{2} i \pi^{-}}{v} \sum_{i j=1,3} V_{j i}^{*}\left[c_{L}^{d_{i}} m_{d_{i}} \bar{d}_{R}^{i}+s_{L}^{d_{i}} M_{D_{i}} \bar{D}_{R}^{i}\right]\left[c_{L}^{u_{j}} u_{L}^{j}+s_{L}^{u_{j}} U_{L}^{j}\right] \\
& -\frac{i \pi^{0}}{v} \sum_{i=1,3}\left[c_{L}^{d_{i}} m_{d_{i}} \bar{d}_{R}^{i}+s_{L}^{d_{i}} M_{D_{i}} \bar{D}_{R}^{i}\right]\left[c_{L}^{d_{i}} d_{L}^{i}+s_{L}^{d_{i}} D_{L}^{i}\right] \\
& +\frac{i \pi^{0}}{v} \sum_{i=1,3}\left[c_{L}^{u_{i}} m_{u_{i}} \bar{u}_{R}^{i}+s_{L}^{u_{i}} M_{U_{i}} \bar{U}_{R}^{i}\right]\left[c_{L}^{u_{i}} u_{L}^{i}+s_{L}^{u_{i}} U_{L}^{i}\right]+\text { h.c. }
\end{aligned}
$$

For all the light quarks, including the $b$, the mixing angles are very small. In principle, one can expect some impact in the precise tests of CKM unitarity, which requires corrections to $V_{u s}$ and $V_{u d}$ not exceeding $1 \%$ and $0.1 \%$, respectively. However, this condition turns out to be easily fulfilled even for small right-handed mixing. Also the tree-level correction to the $b$-quark coupling $g_{L}$, discussed in Section [3.2, turns out to be negligible for $f^{d} \sim M_{D}$.

\section{$5.2 \quad$ Loop effects}

The only significant impact of the light-heavy mixing at low energies arises at the loop level from the top sector 6 . Here the sizable mixing with the heavy partner can lead to non-negligible corrections to the top-induced non-decoupling effects in FCNCs and EWPT. The corrections can be summarised as follows (see the Appendix for more details):

- SM amplitudes which have a finite limit for $x_{t}=m_{t}^{2} / m_{W}^{2} \rightarrow \infty$, such as $\mathcal{A}(b \rightarrow s \gamma)$, are modified in $\left(s=s_{L}^{t}\right)$

$$
F_{S M}\left(x_{t}\right) \rightarrow\left(1-s^{2}\right) F_{S M}\left(x_{t}\right)+s^{2} F_{S M}\left(x_{T}\right), \quad x_{T}=M_{T}^{2} / m_{W}^{2} .
$$

- SM amplitudes which grows linearly with $x_{t}$, such a $\delta T, \mathcal{A}\left(Z \rightarrow \bar{d}^{i} d^{j}\right), \mathcal{A}(\Delta F=2)$, receive a correction which is universal in the gauge-less limit $\left(m_{W} \rightarrow 0\right)$ :

$$
\begin{aligned}
F_{\mathrm{SM}}\left(x_{t}\right) & \rightarrow F_{\mathrm{SM}}\left(x_{t}\right) \times R\left(x_{t}, x_{T}\right) \\
R\left(x_{t}, x_{T}\right) & =1+s^{2}\left[-2+s^{2}\left(\frac{M_{T}^{2}}{m_{t}^{2}}+1\right)+2 \frac{M_{T}^{2}}{M_{T}^{2}-m_{t}^{2}} \ln \left(\frac{m_{T}^{2}}{m_{t}^{2}}\right)+\mathcal{O}\left(\frac{m_{W}^{2}}{m_{t}^{2}}, \frac{m_{W}^{2}}{M_{T}^{2}}\right)\right]
\end{aligned}
$$

The two most interesting phenomenological bounds, arising from $\Delta F=2$ (in particular $\epsilon_{K}$ ) and $Z \rightarrow b \bar{b}$, are summarised in Fig. 1. In the case of $\Delta S=2$ we plot the constraint following from $\left(\epsilon_{K}\right)_{\exp } /\left(\epsilon_{K}\right)_{\mathrm{SM}}=0.92 \pm 0.14$, as derived in [16] from a recent analysis of all $\Delta F=2$ amplitudes in the MFV framework. In the $Z \rightarrow b \bar{b}$ case we show the constraint following from $\left(\delta g_{L}^{b}\right)_{\exp } /\left(\delta g_{L}^{b}\right)_{\mathrm{SM}}=0.86 \pm 0.21$, as derived by the $R_{b}$ measurement [17], where $\delta g_{L}^{b}$ is the deviation

\footnotetext{
${ }^{6}$ For previous related calculations, see [13], 14, 15]
} 


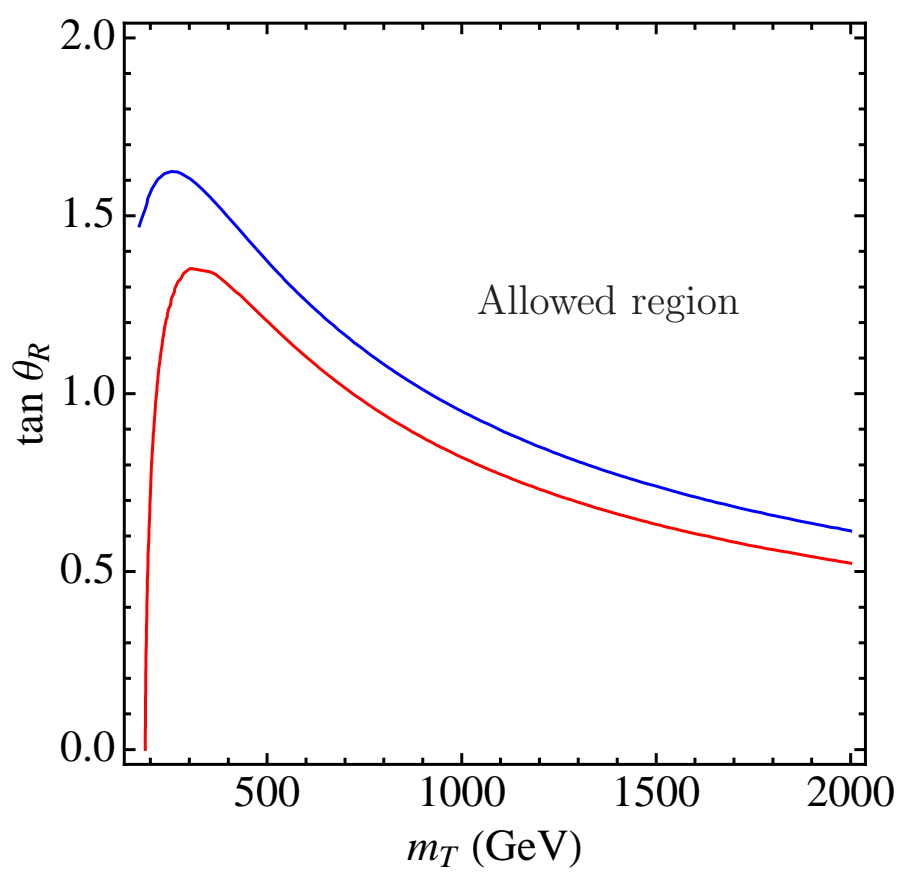

Figure 1: Constraints in the $t_{R}-M_{T}$ plane at $95 \%$ C.L. from $\Delta F=2$ (red curve) and $Z \bar{b} b$ (blue curve).

of $g_{L}^{b}$ from its tree-level SM value. The present constraints form $Z \rightarrow \bar{b} s$ and $b \rightarrow s \gamma$ (see Ref. [18]) are substantially less severe.

As shown in Fig. 1, $t_{R}^{t}=1$ implies a lower bound around $800 \mathrm{GeV}$ for $M_{T}$, but this limit evaporates as soon as $t_{R}^{t} \gtrsim 1.5$. Independently of the value of $M_{T}$ and $t_{R}^{t}$, the correlation of the various loop amplitudes, as described above, implies a small correction to $\delta T$ : a positive contribution which does not exceed $\approx 10 \%$ of $\delta T_{\text {top }}^{\mathrm{SM}}$. This makes it unlikely that a too large contribution to the $S$-parameter can be reconciled with the EWPT by a significant $\delta T$ from composite singlets. Because of the different behaviour in the $m_{t} \rightarrow \infty$ limit, the impact is much smaller in $b \rightarrow s \gamma$, where the positive correction does not exceed $\approx 2 \%$.

\section{Leptons}

The picture described so far for the quarks can be trivially extended to leptons with composites $E_{i}$ and $N_{i}$, one per generation, taking among the elementary leptons also the right-handed neutrinos $\nu_{R_{i}}$. Note that the $N_{i}$ have no interaction at "renormalizable" level. The smallness of the observed neutrino masses can be attributed to a large Majorana mass for the elementary $\nu_{R_{i}}$ 's, $M$, related to the breaking of lepton number and much larger than the compositeness scale of the $N_{i}$, of mass $M_{N}$. This mass among elementary fermions can be present consistently with all the symmetries we have been talking about. Using a notation similar to the one of the quarks, $m_{L}$ has to be 


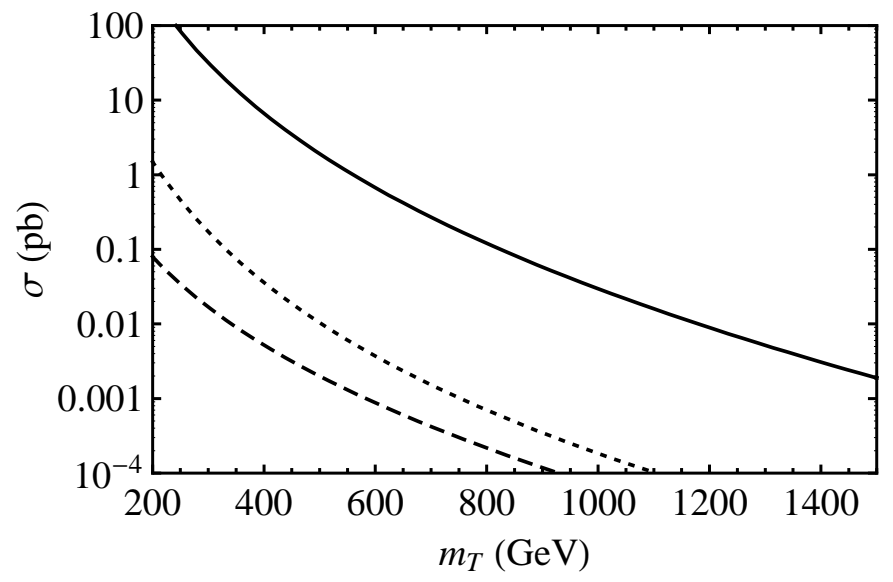

Figure 2: Production cross sections at LHC: for a pair of composite quarks of mass $M$ (full curve); for a singlet $T \bar{t}$ (dashed curve); for a singlet $T \bar{b}$ (dotted curve).

sufficiently smaller than $M_{N}$, so that the light neutrinos are approximately $\nu_{L}+\left(m_{L} / M_{N}\right) N_{L}$ and have mass

$$
m_{\nu}^{\text {light }} \approx\left(\frac{m_{L}}{M_{N}}\right)^{2} \frac{m_{R}^{2}}{M} .
$$

It may be interesting to study the phenomenology and the cosmology $7^{7}$ of the neutrino sector so extended, which is outside the scope of this work.

\section{Collider Phenomenology}

At the LHC, the pair production of any composite quark proceeds by gluon-gluon fusion. Single production, on the contrary, in association with an elementary quark is a weak Drell Yan process. In Fig. 2 we give both the pair-production cross section for a generic composite quark and the $T \bar{t}$ and $T \bar{b}$ cross sections in the case of composite singlets, using the currents in (5.8). The single production cross sections are reduced by the small mixing angles (5.5) , making all of them negligibly small but the one of the $T$.

The three singlets, of charge $2 / 3$ or $-1 / 3$, have splittings

$$
\frac{M_{i}-M_{j}}{\langle M\rangle} \sim\left\{\frac{m_{i}-m_{j}}{v}, \frac{\left(m_{i}^{2}-m_{j}^{2}\right)}{\left\langle M^{2}\right\rangle}\right\},
$$

respectively in the $\mathrm{PC}$ and $\mathrm{PB}$ case, where $m_{i}$ are the masses of the corresponding elementary quarks. Except the partner of the top in the PC case, all the other composites of given charge are highly degenerate.

\footnotetext{
${ }^{7}$ As an example, the composite $N$ partner of a massless, or quasi massless neutrino, might be a Dark Matter candidate for suitable values of its non renormalizable interactions.
} 
The composite singlets have a narrow width, which is most easily computed by means of (5.9). The dominant decay of $U_{i}$ or $D_{i}$ is in the corresponding light state, $u_{i}$ or $d_{i}$ plus a $W$ or a $Z$, with

$$
\Gamma\left(U_{i} \rightarrow d_{i}+W\right) \approx 2 \Gamma\left(U_{i} \rightarrow u_{i}+Z\right) \approx \frac{1}{16 \pi}\left(s_{L}^{u_{i}}\right)^{2} \frac{M_{U_{i}}^{3}}{v^{2}}
$$

and, analogously,

$$
\Gamma\left(D_{i} \rightarrow u_{i}+W\right) \approx 2 \Gamma\left(D_{i} \rightarrow d_{i}+Z\right) \approx \frac{1}{16 \pi}\left(s_{L}^{d_{i}}\right)^{2} \frac{M_{D_{i}}^{3}}{v^{2}}
$$

In view of Section 5.1, the total widths of $U_{i}$, and similarly for $D_{i}$, can be written as

$$
\left.\Gamma_{T o t}\left(U_{i}\right)\right|_{\mathrm{PC}} \approx \frac{3}{32 \pi}\left(\frac{m_{u_{i}} M_{U}^{2}}{v f^{u}}\right) \approx 0.1 \mathrm{MeV}\left(\frac{m_{u_{i}}}{\mathrm{MeV}}\right)\left(\frac{M_{U}^{2}}{f^{u} \mathrm{TeV}}\right)
$$

or

$$
\left.\Gamma_{\text {Tot }}\left(U_{i}\right)\right|_{\mathrm{PB}} \approx \frac{3}{32 \pi}\left(\frac{m_{u_{i}} M_{U^{2}}^{2} M_{U_{i}}}{v^{2}\left(f^{u}\right)^{2}}\right) \approx 0.5 \mathrm{eV}\left(\frac{m_{u_{i}}}{\mathrm{MeV}}\right)^{2}\left(\frac{M_{U^{2}}^{2} M_{U_{i}}}{\left(f^{u}\right)^{2} \mathrm{TeV}}\right) .
$$

Taking the last factor in the r.h.s. of these equations equal to unity, these widths range from about $0.1 \mathrm{MeV}$ for $U_{1}$ to about $200 \mathrm{MeV}$ for $D_{3}$ in the PC case, whereas they go from a fraction of $1 \mathrm{eV}$ for $U_{1}$ to about $1 \mathrm{MeV}$ for $D_{3}$ in the PB case.

All this is based on the mass mixings described in Sect. 4. One can ask if these decay properties could be changed by the presence of higher dimensional operators consistent with the symmetries. There is no such operator at any relevant level in the Parity Conserving case. In the Parity Breaking case the operator

$$
\Delta L=\frac{f^{u}}{\Lambda^{3}}\left(\bar{U}_{R}^{i} \gamma_{\mu} u_{R}^{i}\right) \Sigma_{j}\left(\bar{q}^{j} \gamma_{\mu} q^{j}\right),
$$

if present, would make the decay of $U_{1}$ and $U_{2}$ dominated by the modes

$$
U_{i} \rightarrow u_{i}+\bar{f} f
$$

where $f$ is any elementary fermion, although still with a small width of about $0.1 \mathrm{MeV}$. Similar considerations hold for the $D_{i}$.

For several aspects (production cross-sections and leading decay modes into $W+q$ ) the phenomenology of these heavy states at colliders is quite similar to that of sequential fermion families within the SM (see e.g. [19]). Beside the narrow decay widths, an important difference is the large neutral-current branching fraction into $Z$ bosons, as indicated in eqs.(7.2)-(7.3). The $Z+q$ final state, which can have a non-negligible branching fraction also for sequential quarks under specific circumstances [19], is definitely the most interesting one for searches at hadron colliders. According to a recent CDF study [20], a bound of about $270 \mathrm{GeV}$ can be set on the mass of the partner of the $b$ quark, assuming $\operatorname{BR}(B \rightarrow b Z)=100 \%$. 


\section{Summary and Conclusions}

A consistent description of

- unitarity in $W W$ scattering,

- the EWPT,

- fermion masses and flavour physics,

is greatly eased in the SM by the presence of a Higgs boson, which makes its search, perhaps in a supersymmetric realm, a primary task of the LHC. The competitive view, based on a strong dynamics, has definitely a harder time in achieving the same goals, at least when one tries to come to concrete models. However we do not forget, on one side, the scanty direct experimentation at the Fermi scale or above it and, on the other side, that the SM itself is likely to be an effective theory. Altogether, this still motivates the study of possible generic features of strongly interacting theories of Electroweak Symmetry Breaking by making the least possible reference to explicit models.

Along these lines, in this work we have analyzed the properties and the constraints on possible composite fermions that might result quite naturally from the strong dynamics. As already pointed out, this is made possible at all by focusing on two approximate symmetries:

- a chiral $S U(2)_{L} \times S U(2)_{R}$ that breaks down to its diagonal subgroup,

- a symmetry $G_{f}$ that enlarges the flavour symmetry of the SM, in absence of the Higgs doublet, to include the flavour symmetry of the composite fermions themselves.

While the first is a widely accepted feature of strong EWSB, the second one is meaningful and useful if one takes the view that the masses of the standard elementary fermions only arise from their mixing with the composite fermions. We find this assumption coherent with the picture that the standard fermions do not participate in the strong dynamics, which is the source of EWSB, whereas their masses do break the electroweak symmetry. We hope that the multiplicity of the composite fermions needed to this purpose be explained by the strong dynamics.

Our results can be summarized as follows. We consider Singlets, Doublets and Triplets under the custodial $S U(2)_{L+R}$. In a truly strongly interacting theory of EWSB we find that Doublets and Triplets are faced with difficulties, although a generic analysis like ours cannot exclude them. On the other hand the Singlets, whose composite nature is admittedly hidden, may nevertheless play an important role. This is in particular the case in flavour physics, where we show that it becomes pretty natural to keep the CKM picture of the SM with MFV. Under this assumption, we can specify the fine structure of the spectrum of the composite fermions, three of charge $2 / 3, U_{i}$, and three of charge $1 / 3, D_{i}$, and their decay properties, relevant to the search at the LHC. Equally specified is their loop contribution to the $T$-parameter in the EWPT and to several flavour observables. The correlation between these contributions excludes a significant effect of the Singlets in the T-parameter. At the same time the non observation of significant deviation in flavour physics from the SM does not set any strict lower bound on the their masses. The search for the heavy quarks, with a 30\% branching ratio into an ordinary quark and a $Z$-boson, might be feasible up to significantly large values of their masses even in the early stage of the LHC. Whereas we have concentrated on coloured states, all the picture can be naturally extended to 
leptons. Several aspects of the phenomenology of these composites, if they exist at all, deserve further study.

\section{Acknowledgements}

We thank Slava Rychkov for useful discussions in the early stage of this work and Paolo Lodone for help in checking the loop calculations. R.B. was partially supported by the EU under RTN contract MRTN-CT-2004-503369 and by MIUR under the contract PRIN-2006022501. G.I. was partially supported by the EU under RTN contract MTRN-CT-2006-035482.

\section{A Heavy-fermion effects in FCNCs}

Following the notation of Buras [21] we write the leading electroweak contributions to $b \rightarrow s$ FCNC transitions in the SM as follows:

$$
\begin{aligned}
\mathcal{H}(\Delta B=2) & =\lambda_{t} \frac{G_{\mathrm{F}}^{2} m_{W}^{2}}{4 \pi^{2}} S_{0}\left(x_{t}\right)\left(\bar{b}_{L} \gamma^{\mu} s_{L}\right)^{2} \\
\mathcal{H}(b \rightarrow s \nu \bar{\nu}) & =\lambda_{t} \frac{G_{\mathrm{F}}}{\sqrt{2}} \frac{2 \alpha}{\pi \sin ^{2} \Theta_{\mathrm{W}}}\left[C_{0}\left(x_{t}\right)-4 B_{0}\left(x_{t}\right)\right]\left(\bar{s}_{L} \gamma^{\mu} d_{L}\right)\left(\bar{\nu} \gamma_{\mu} \nu\right) \\
\mathcal{H}(b \rightarrow s \mu \bar{\mu}) & =\lambda_{t} \frac{G_{\mathrm{F}}}{\sqrt{2}} \frac{2 \alpha}{\pi \sin ^{2} \Theta_{\mathrm{W}}}\left[C_{0}\left(x_{t}\right)+B_{0}\left(x_{t}\right)\right]\left(\bar{s}_{L} \gamma^{\mu} d_{L}\right)\left(\bar{\mu} \gamma_{\mu} \mu\right) \\
\mathcal{H}(b \rightarrow s \gamma) & =\lambda_{t} \frac{G_{\mathrm{F}}}{\sqrt{2}} \frac{e}{8 \pi^{2}} D_{0}^{\prime}\left(x_{t}\right) m_{b} \bar{b}_{R} \sigma^{\mu \nu} F_{\mu \nu} s_{L} \\
\mathcal{H}(b \rightarrow s G) & =\lambda_{t} \frac{G_{\mathrm{F}}}{\sqrt{2}} \frac{g_{s}}{8 \pi^{2}} E_{0}^{\prime}\left(x_{t}\right) m_{b} \bar{b}_{R} \sigma^{\mu \nu} T^{a} G_{\mu \nu}^{a} s_{L}
\end{aligned}
$$

where $\lambda_{t}=V_{t b}^{*} V_{t s}$ and $x_{t}=m_{t}^{2} / m_{W}^{2}$. The corresponding terms for $b \rightarrow d$ and $s \rightarrow d$ transitions -in the limit where we can neglect the charm quark mass- are obtained by replacement of the CKM factor and by $m_{b} \rightarrow m_{s}$. The explicit expressions of the loop functions are:

$$
\begin{array}{rlrl}
S_{0}(x) & =\frac{4 x-11 x^{2}+x^{3}}{4(1-x)^{2}}-\frac{3 x^{3} \ln x}{2(1-x)^{3}}, & S_{0}(x) \stackrel{x \rightarrow \infty}{\longrightarrow} \frac{x}{4} \\
C_{0}(x)=\frac{x}{8}\left[\frac{x-6}{x-1}+\frac{3 x+2}{(x-1)^{2}} \ln x\right], & C_{0}(x) \stackrel{x \rightarrow \infty}{\longrightarrow} \frac{x}{8} \\
B_{0}(x)=\frac{1}{4}\left[\frac{x}{1-x}+\frac{x \ln x}{(x-1)^{2}}\right], & B_{0}(x) \stackrel{x \rightarrow \infty}{\longrightarrow}-\frac{1}{4} \\
D_{0}^{\prime}(x) & =-\frac{\left(8 x^{3}+5 x^{2}-7 x\right)}{12(1-x)^{3}}+\frac{x^{2}(2-3 x)}{2(1-x)^{4}} \ln x, & D_{0}^{\prime}(x) \stackrel{x \rightarrow \infty}{\longrightarrow} \frac{2}{3} \\
E_{0}^{\prime}(x) & =-\frac{x\left(x^{2}-5 x-2\right)}{4(1-x)^{3}}+\frac{3}{2} \frac{x^{2}}{(1-x)^{4}} \ln x, & E_{0}^{\prime}(x) \stackrel{x \rightarrow \infty}{\longrightarrow} \frac{1}{4}
\end{array}
$$


In the cases where the $x \rightarrow \infty$ limit is finite, the modification of the coefficient functions due the addition of the heavy state is

$$
F\left(x_{t}\right) \rightarrow c^{2} F\left(x_{t}\right)+s^{2} F\left(x_{T}\right)=F\left(x_{t}\right)+s^{2}\left[F\left(x_{T}\right)-F\left(x_{t}\right)\right], \quad F=B_{0}, D_{0}^{\prime}, E_{0}^{\prime}
$$

where $x_{T}=M_{T}^{2} / m_{W}^{2}$. As expected, the correction vanishes for $s=0$ or $x_{t}=x_{T}$ (which corresponds to setting $m_{L}=m_{R}$ and $M_{Q}=0$ in the mass matrix). In this specific case the correction vanishes also for $M_{T}, m_{t} \gg m_{W}$. Note, however, that the $x_{t} \rightarrow \infty$ limit is not necessarily a good numerical approximation for the physical top-quark mass. For instance in the $b \rightarrow s \gamma$ case $\left[D_{0}^{\prime}(\infty)-D_{0}^{\prime}\left(x_{t}^{\mathrm{phys}}\right)\right] \approx 0.7 D_{0}^{\prime}\left(x_{t}^{\mathrm{phys}}\right)$.

For the amplitudes where the $x \rightarrow \infty$ limit is not finite $(\Delta F=2$ box and $Z$ penguin) we cannot express the result using only SM loop functions. The result can be written in the following general form:

$$
\begin{aligned}
F\left(x_{t}\right) \rightarrow & {\left[c^{4} \bar{F}\left(x_{t}, x_{t}\right)+s^{4} \bar{F}\left(x_{T}, x_{T}\right)+2 c^{2} s^{2} \bar{F}\left(x_{t}, x_{T}\right)\right]-c^{2} \Delta F\left(x_{t}\right)-s^{2} \Delta F\left(x_{T}\right) } \\
& =F\left(x_{t}\right)+s^{2}\left[\left(s^{2}-2\right) \bar{F}\left(x_{t}, x_{t}\right)+s^{2} \bar{F}\left(x_{T}, x_{T}\right)+2 c^{2} \bar{F}\left(x_{t}, x_{T}\right)\right] \\
& -s^{2}\left[\Delta F_{S}\left(x_{T}\right)-\Delta F_{S}\left(x_{t}\right)\right],
\end{aligned}
$$

where

$$
\Delta F(x) \equiv \bar{F}(x, x)-F(x), \quad \frac{\Delta_{F}(x)}{F(x)} \stackrel{x \rightarrow \infty}{\longrightarrow} 0, \quad F=S_{0}, C_{0} .
$$

As expected, also in this case the correction vanishes for $s=0$ or $x_{t}=x_{T}$. The leading functions $\bar{F}(x, x)$ for the two relevant cases can be identified by the explicit calculation of the diagrams with two different heavy propagators. In the limit $m_{W} \rightarrow 0$ the result is particularly simple and universal,

$$
\frac{\bar{F}\left(x_{T}, x_{T}\right)}{\bar{F}\left(x_{t}, x_{t}\right)}=\frac{M_{T}^{2}}{m_{t}^{2}}, \quad \frac{\bar{F}\left(x_{T}, x_{t}\right)}{\bar{F}\left(x_{t}, x_{t}\right)}=\frac{M_{T}^{2}}{M_{T}^{2}-m_{t}^{2}} \ln \left(\frac{m_{T}^{2}}{m_{t}^{2}}\right),
$$

leading to the function $R\left(x_{t}, x_{T}\right)$ in Eq. (5.11). The reason for this universality can be understood by the fact that in the gaugeless limit the only UV and IR finite integral with two heavy propagators, which exhibit the right grow for $m_{1}=m_{2} \rightarrow \infty$, is

$$
\int d l^{2} \frac{m_{1}^{2} m_{2}^{2}}{\left(l^{2}+m_{1}^{2}\right)\left(l^{2}+m_{2}^{2}\right)}=\frac{m_{1}^{2} m_{2}^{2}}{m_{1}^{2}-m_{2}^{2}} \ln \left(\frac{m_{1}^{2}}{m_{2}^{2}}\right)
$$

The complete expressions of the loop functions, necessary to evaluate also the subleading terms, are

$$
\begin{aligned}
\bar{S}_{0}\left(x_{1}, x_{2}\right) & =-\frac{3 x_{1} x_{2}}{4\left(1-x_{1}\right)\left(1-x_{2}\right)}+\left[\frac{x_{1} x_{2}\left(4-8 x_{1}+x_{1}^{2}\right)}{4\left(1-x_{1}\right)^{2}\left(x_{1}-x_{2}\right)} \ln \left(x_{1}\right)+\left[x_{1} \leftrightarrow x_{2}\right]\right], \\
\Delta S_{0}(x) & =0
\end{aligned}
$$

for the box diagram, and

$$
\begin{aligned}
\bar{C}_{0}\left(x_{1}, x_{2}\right) & =\frac{x_{1}^{2}\left(x_{2}-1\right)}{8\left(x_{1}-x_{2}\right)\left(x_{1}-1\right)} \ln \left(x_{1}\right)+\left[x_{1} \leftrightarrow x_{2}\right], \\
\Delta C_{0}(x) & =-\frac{5}{8} \frac{x(1-x+x \ln (x))}{(x-1)^{2}},
\end{aligned}
$$


for the $Z$ penguin.

The general decomposition in (A.12) applies also the the flavour-conserving non-decoupling effects in $\delta T$ and $Z \rightarrow \bar{b} b$. Since the approximate expression in Eq. (5.11) turns out to be an excellent numerical approximation for the $Z \rightarrow \bar{b} s$ penguin, we have used it in Fig. 1 to estimate the bound derived from $Z \rightarrow \bar{b} b$.

\section{References}

[1] C. Csaki, C. Grojean, H. Murayama, L. Pilo and J. Terning, Phys. Rev. D 69, 055006 (2004) arXiv:hep-ph/0305237.

[2] R. Foadi, S. Gopalakrishna and C. Schmidt, JHEP 0403 (2004) 042 arXiv:hep-ph/0312324.

[3] H. Georgi, Phys. Rev. D 71 (2005) 015016 [arXiv:hep-ph/0408067].

[4] R. Barbieri, G. Isidori, V. S. Rychkov and E. Trincherini, arXiv:0806.1624 [hep-ph].

[5] D. B. Kaplan, Nucl. Phys. B 365 (1991) 259.

[6] S. R. Coleman et. al. Phys. Rev. 177, 2239, 2247 (1969); C.G. Callan, et. al. Phys. Rev. 177 (1969) 2247.

[7] R. S. Chivukula, B. Coleppa, S. Di Chiara, E. H. Simmons, H. J. He, M. Kurachi and M. Tanabashi, Phys. Rev. D 74 (2006) 075011 arXiv:hep-ph/0607124.

[8] K. Agashe, R. Contino, L. Da Rold and A. Pomarol, Phys. Lett. B 641 (2006) 62 arXiv:hep-ph/0605341.

[9] G. D'Ambrosio, G. F. Giudice, G. Isidori and A. Strumia, Nucl. Phys. B 645 (2002) 155 arXiv:hep-ph/0207036.

[10] M. Antonelli et al. [FlaviaNet Working Group on Kaon Decays], arXiv:0801.1817 [hep-ph].

[11] S. Davidson, G. Isidori and S. Uhlig, Phys. Lett. B 663 (2008) 73 [arXiv:0711.3376 [hep-ph]].

[12] R. Contino, T. Kramer, M. Son and R. Sundrum, JHEP 0705 (2007) 074 arXiv:hep-ph/0612180.

[13] M. S. Carena, E. Ponton, J. Santiago and C. E. M. Wagner, Phys. Rev. D 76, 035006 (2007) arXiv:hep-ph/0701055.

[14] R. Barbieri, B. Bellazzini, V. S. Rychkov and A. Varagnolo, Phys. Rev. D 76 (2007) 115008 arXiv:0706.0432 [hep-ph]].

[15] P. Lodone, arXiv:0806.1472 [hep-ph].

[16] M. Bona et al. [UTfit Collaboration], JHEP 0803 (2008) 049 [arXiv:0707.0636 [hep-ph]].

[17] The LEP Collaborations, Phys. Rept. 427 (2006) 257 arXiv:hep-ex/0509008, and on-line update at http://lepewwg.web.cern.ch/LEPEWWG/

[18] T. Hurth et al., arXiv:0807.5039 [hep-ph].

[19] P. H. Frampton, P. Q. Hung and M. Sher, Phys. Rept. 330 (2000) 263 arXiv:hep-ph/9903387. 
[20] T. Aaltonen et al. [CDF Collaboration], Phys. Rev. D 76 (2007) 072006 arXiv:0706.3264 [hep-ex]].

[21] A. J. Buras, Weak Hamiltonian, CP violation and rare decays, arXiv:hep-ph/9806471. 